\title{
Molecular springs and muscles: Progress toward augmented electromechanical actuation*
}

\author{
Adah Almutairi, Kunsang Yoon, Fook Tham, and Michael J. Marsella ${ }^{\ddagger}$ \\ Department of Chemistry, University of California, Riverside, CA 92521-0403, USA
}

\begin{abstract}
The application of 3,3'-diphenyl-2,2'-bithiophene as a helical scaffold capable of electrochemical polymerization to yield the corresponding polythiophene is reported. One unique feature of this monomer is its theoretically predicted (DFT) ability to mimic redoxstimulated contraction and expansion. This ability, coupled with traditional electromechanical actuation properties of bulk, redox-active conjugated polymers (CPs), yields a polymeric "molecular muscle" capable of both contraction and expansion.
\end{abstract}

Keywords: electromechanical actuation; polythiophene; helicity; diphenylbithiophene; molecular muscle.

The generation of motion, referred to also as actuation, results from free-energy transduction caused by a chemical, electronic, or electromagnetic stimulus [1]. An elegant actuating system developed by Nature is skeletal muscle. Muscles are composed of 1D filaments, bound together by van der Waals (VDW) interactions into bundles of fibers. The filaments, containing copper(II/I) centers and phosphate ligands, are set into motion by chemical signal, which initiates a reversible actuation (chemomechanical actuation).

Actuators composed of conjugated polymers (CPs) serve as non-natural surrogates of biological muscle. The systems act as electromechanical actuators (EMAs, a.k.a. "molecular muscles") via redoxinduced counterion adsorption and expulsion. For example, oxidation of a bulk CP sample requires an influx of charge-balancing counterions and solvating species. The intercalated species have a defined volume, which results in the oxidized $\mathrm{CP}$ to have a volume greater than the neutral $\mathrm{CP}$. This reversible volume change can be utilized to perform work. Note that the mechanism invoked by traditional CP-EMAs relies on ion and solvent intercalation, thus is not intrinsic to CP strands [2]. Often, this mechanism leads to degradation of the life and rate of faradaic conducting polymer actuators [2]. Recent efforts in the field of molecular actuators have focused on investigating and expanding upon the properties and behavior of electrolytes [3].

A different approach would be to augment the actuation resulting from redox-induced ion-intercalation (bulk actuation) with CPs that are capable of intrinsic actuation [4-10]. Specifically, if each repeat unit of a polymer was capable of redox-induced conformational change that results in a change in volume and/or length, then intrinsic actuation could act to augment bulk actuation. Preferably, intrinsic actuation would be active in the "light-doping regime" of the polymer, where "light doping" implies that the polymer is oxidized to a state less than that corresponding to the observed peak anodic current $\left(I_{\mathrm{p}, \mathrm{a}}\right.$, as determined from cyclic voltammetry). This strategy may help remedy degradation problems resulting from high CP oxidation states ("heavy doping").

\footnotetext{
*Paper based on a presentation at the $11^{\text {th }}$ International Symposium on Novel Aromatic Compounds (ISNA-11), St. John's, Newfoundland, Canada, 14-18 August 2005. Other presentations are published in this issue, pp. 685-888.

¥Corresponding author
} 
In this report, we communicate the properties of ortho-diarylbithiophenes (o-DABTs) and corresponding polymers. Furthermore, we illustrate their potential as "spring-like" monomers for intrinsic CP-EMAs. Ideally, such intrinsic actuators may provide more efficient CP-EMAs by strategically coupling the compression of molecular springs with bulk CP-EMA contraction (vide infra).

We have previously reported that sexithiophenes coupled at the 2,3-positions reliably exhibit helical motifs in the solid state (see compound 1, Fig. 1). The driving force for this conformation is a combination of steric and torsional strain resulting from the "ortho-connectivity" of the thiophene rings [11]. In this study, we investigate compounds $\mathbf{2}$ and $\mathbf{3}$ for their ability to adopt a helical conformation. Specifically, we dissected the parent sexithiophene, compound 1, into tetraaryl analogs of two categories differentiated by their central bithiophene unit (Fig. 1); compound 2 corresponds to a 2,2-bithiophene core and compound $\mathbf{3}$ corresponds to a 3,3'-bithiophene core. In both cases, the bithiophene core is terminated with phenyl rings to achieve the minimum number of rings to complete one turn of a helix. The rationale for the inclusion of terminal phenyl rings is to limit the number of reactive $\alpha$-thienyl sites to two. This mandates that electrochemical polymerization would occur specifically through the bithiophene unit [12].
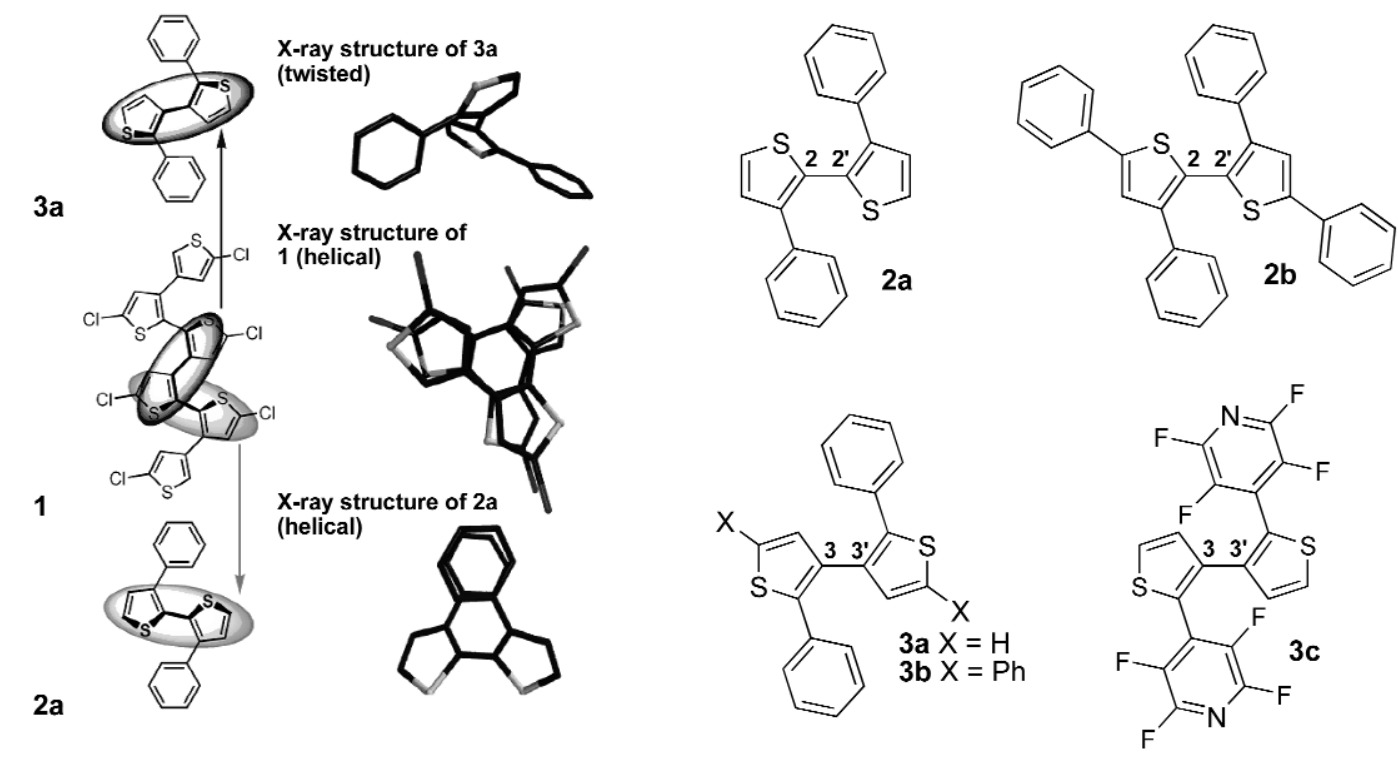

Fig. 1 Parent sexithiophene, compound 1 can be dissected into both 2,2'- and 3,3'-bithienyl fragments. Compounds $\mathbf{2}$ and $\mathbf{3}$ reflect the two regioisomeric bithienyl cores. Illustrations for all compounds $\mathbf{1}, \mathbf{2 a}, \mathbf{b}$, and 3a-c, and X-ray structures of compounds $\mathbf{1}, \mathbf{2} \mathbf{a}$, and $\mathbf{3 a}$ (hydrogens omitted) are shown. Note only type $\mathbf{2}$ compounds adopt helical structures analogous to compound $\mathbf{1}$.

Figure 1 shows the four derivatives reported herein, compounds $\mathbf{2 a}$ and $\mathbf{3 a}$ bear no $\alpha$-thienyl substituents, compounds $\mathbf{2} \mathbf{b}$ and $\mathbf{3 b}$ have phenyl substituents at both $\alpha$-thienyl positions, and compound $\mathbf{3 c}$ has chlorines at the two $\alpha$-theinyl positions as well as a perfluoropyridyl in place of the phenyl groups in 3a. Merck molecular force field (MMFF) calculations, which have proven successful at predicting the solid-state structure of ortho-oligothiophenes, predict that compounds $\mathbf{2}$ and $\mathbf{3}$ yield two distinctly different packing motifs. Compounds $\mathbf{2 a}, \mathbf{b}$ are predicted to adopt ahelical conformation analogous to compound $\mathbf{1}$. However, compounds $\mathbf{3 a - c}$ prefer a more open structure, despite their peripheral constitutional differences. These predictions were supported experimentally by X-ray analysis of compounds $\mathbf{2 a}, \mathbf{2 b}, \mathbf{3 a}$, and $\mathbf{3 c}$. The similarity of features within a class (i.e., types $\mathbf{2}$ and $\mathbf{3}$ ) as well as correlation with MMFF predicted structures are both communicated graphically in Fig. 2. 

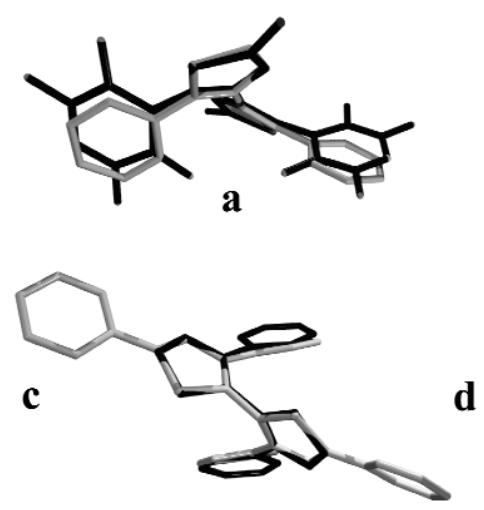

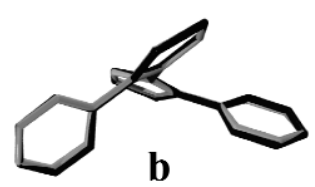

d

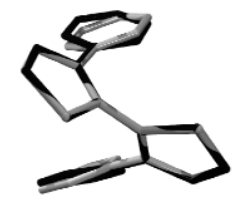

Fig. 2 Overlaid images of: (a) the X-ray structures of compounds 3a (gray) and 3c (black); (b) the MMFF lowestenergy conformer of 3a (gray) and the X-ray structure of 3a; (c) the X-ray structures of compounds $\mathbf{2 a}$ (black) and $\mathbf{2 b}$ (gray); and (d) the MMFF lowest-energy conformer of $\mathbf{2 a}$ (gray) and the X-ray structure of $\mathbf{2 b}$.

Note that compound $\mathbf{2 b}$ illustrates that further aromatic substitution at the two $\alpha$-thienyl positions does not preclude helical conformation; the backbones of compounds $\mathbf{2 a}$ and $\mathbf{2 b}$ adopt nearly identical helical conformation. This observation adds credibility to our design of a helical repeat unit in the corresponding polymer. In agreement with MMFF calculations, neither 3,3'-bithienyl compounds 3a nor 3b adopt a tight helical conformation.

The UV-vis absorption spectra of compounds $\mathbf{2 a}, \mathbf{3 a}$ and $\mathbf{2} \mathbf{b}, \mathbf{3 b}$ are reported in Fig. 3. The major feature is a slightly lower-energy onset of absorption for the 2,2'-bithienyl systems, compounds $\mathbf{2 a}, \mathbf{b}$, and a greater intensity of absorption in the $>250 \mathrm{~nm}$ UV absorption window for the 3,3'-bithiophienyl systems, compounds 3a,b. The integrative absorption of compound 3a relative to compound $\mathbf{2 a}$ may be attributed to the pseudo-high concentration of phenylthiophene moieties in compound $\mathbf{3}$ (two non-interacting chromophores) vs. a single, fully conjugated diphenylbithiophene moiety in compound $\mathbf{2}$.
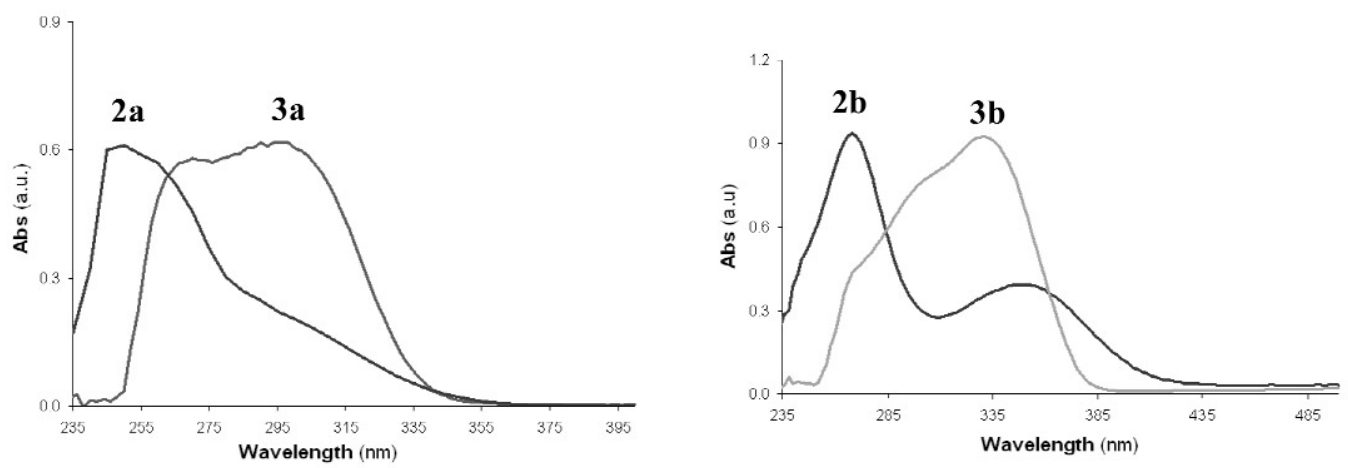

Fig. 3 UV-vis spectra of compounds $\mathbf{2 a}$ and $\mathbf{3 a}$ (left); $\mathbf{2 b}$ and $\mathbf{3 b}$ (right).

The possibility of intrinsic actuation in compound 2a can be envisioned as follows. Compound 2a may be considered a redox-active spring. Upon oxidation, the backbone dihedral angles decrease in value, likely in an effort to optimize $\mathrm{p}_{\mathrm{z}}$-orbital overlap (and thus delocalization of charge [13]). Specifically, BLYP/6-31G[d] geometry optimization of $\mathbf{2 a}^{1+}$ yields a $10 \%$ compression of the long helical axis. Electrochemical polymerization at the free $\alpha$-positions of compound $2 \mathbf{a}$ yields poly(2a), a system having the helical long axis of monomer $\mathbf{2 a}$ orthogonal to the long axis of poly(2a) (see representation in Fig. 4). The phenyl groups of monomer 2a serving as side-chains that enforce interchain 

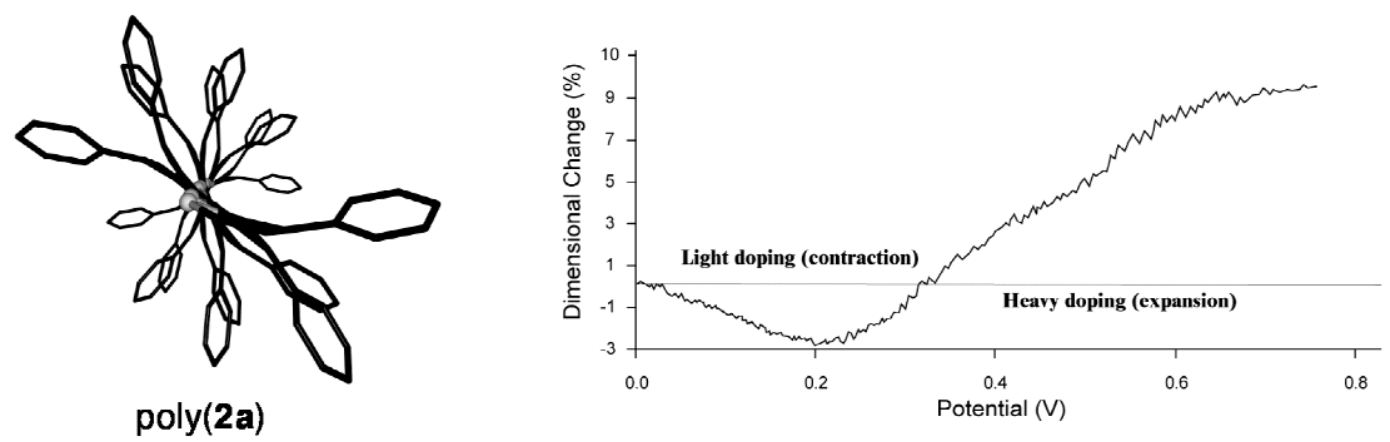

Fig. 4 Left: snapshot of a molecular dynamics simulation of poly(2a), as viewed down the long polymer axis. Right: plot of CP-EMA dimensional change in poly(2a) as a function of oxidation state (see text).

spacing. If oxidation of poly(2a) results in contraction of the helical repeat units, then the effective spacing between polymer chains would necessarily decrease [14]. Given that CPs typically undergo a contraction of ca. 0.5-1.0\% upon light doping (a bulk process), there exists a synergy between bulk and intrinsic processes. As such, EMA in the contraction mode should be observed until a critical point is reached, corresponding to the condition whereby effects of ion intercalation cause bulk expansion to outweigh contraction.

Poly(2a) is a conductive polymer absorbing at $420 \mathrm{~nm}$. Our molecular dynamic simulations (shown on left in Fig. 4) explain this short effective conjugation length to be due to a spiraling backbone [12]. Figure 4 also reports the real-time EMA response to an $80-\mu \mathrm{m}$-thick film of poly(2a), measured according to our previously reported cyclic-voltammetry/laser-micrometry analytical technique [15]. In this example, contraction reaches a maximum of $3 \%$ at $0.2 \mathrm{~V}$ (within the "light-doping" regime), and a maximum expansion of $9 \%$. Recalling that CP-EMAs typically undergo a bulk compression of ca. $0.5-1.0 \%$, we emphasize that poly(2a) achieves a three-fold increase in observed compression. This promising observation should be viewed with optimistic caution, as we are unable to elucidate the contribution of each individual process (intrinsic vs. bulk) at this time.

Advantages to actuation in the light-doping regime include lower voltage requirements, higherenergy efficiencies, and faster response times (given that the flux of counterions will be at a minimum). In terms of energy input per unit volume per percent dimensional change $\left(\% \Delta_{\mathrm{D}}\right)$, poly $(\mathbf{2 a})$ required $1.14 \mathrm{~J} / \mathrm{cm}^{3}$ in the heavy-doping regime, while the light-doping regime required only $6.16 \times 10^{-03} \mathrm{~J} / \mathrm{cm}^{3}$. Specifically, an energy input for a $1 \% \Delta_{\mathrm{D}}$ expansion in the traditional heavy-doping regime is three orders of magnitude greater than the energy required for a $1 \% \Delta_{\mathrm{D}}$ contraction in the light-doping regime. Given the calculated lower-energy requirements and promising initial results, we propose the "augmented" approach of coupling intrinsic and bulk EMA properties in the light-doping regime requires further investigation.

\section{REFERENCES}

1. D. W. Urry. Angew. Chem., Int. Ed. Engl. 32, 819 (1993).

2. R. H. Baughman. Synth. Met. 78, 339 (1996).

3. J. Ding, D. Z. Zhou, G. Spinks, G. Wallace, S. Forsyth, M. Forsyth, D. MacFarlane. Chem. Mater. 15, 2392 (2003).

4. M. J. Marsella, R. J. Reid. Macromolecules 32, 5982 (1999).

5. M. J. Marsella, R. J. Reid, S. Estassi, L. S. Wang. J. Am. Chem. Soc. 124, 12507 (2002).

6. M. C. Jimenez, C. Dietrich-Buchecker, J. P. Sauvage. Angew. Chem., Int. Ed. 39, 3284 (2000). 
7. B. Jousselme, P. Blanchard, E. Levillain, J. Delaunay, M. Allain, P. Richomme, D. Rondeau, N. Gallego-Planas, J. Roncali. J. Am. Chem. Soc. 125, 1363 (2003).

8. K. J. Lin, S. J. Fu, C. Y. Cheng, W. H. Chen, H. M. Kao. Angew. Chem., Int. Ed. 43, 4186 (2004).

9. H. H. Yu, T. M. Swager. IEEE J. Oceanic Eng. 29, 692 (2004).

10. H. H. Yu, B. Xu, T. M. Swager. J. Am. Chem. Soc. 125, 1142 (2003).

11. M. J. Marsella, K. Yoon, A. Almutairi, S. K. Butt, F. S. Tham. J. Am. Chem. Soc. 125, 13928 (2003).

12. A. Almutairi, F. S. Tham, M. J. Marsella. Tetrahedron 60, 7187 (2004).

13. R. Herges, D. Geuenich. J. Phys. Chem. A 105, 3214 (2001).

14. M. J. Winokur, P. Wamsley, J. Moulton, P. Smith, A. J. Heeger. Macromolecules 24, 3812 (1991).

15. M. J. Marsella, R. J. Reid, M. T. McCormick. Synth. Met. 118, 171 (2001). 